\title{
Hermann-Altrock-Stipendium des Deutschen Sportbundes
}

1. Das Hermann-Altrock-Stipendium wird alljährlich in Höhe von DM 5000, - an einen Sportstudierenden oder einen Doktoranten aus der Bundesrepublik Deutschland verliehen.

2. Das Stipendium soll es dem Empfänger ermöglichen, nach bestandener sportpraktischer Prüfung sein Studium für zwei Semester im Ausland fortzusetzen bzw. eine thematisch wichtige sportwissenschaftliche Dissertation anzufertigen.

3. Vorschläge für die Verleihung mit Referenzen der Hochschullehrer und einem handschriftlichen Lebenslauf des Bewerbers (mit Lichtbild), in dem dieser u. a. die beabsichtigte Verwendung des Stipendiums darlegt, sind durch die Institute für Leibesübungen und die Pädagogischen Hochschulen jeweils zum 1. Oktober an den

Deutschen Sportbund, Bundesausschuß für Wissenschaft und Bildung, Otto-Fleck-Schneise 12, 6000 Frankfurt/Main 71,

zu richten.

4. Beurteilungskriterien für die Vergabe des Stipendiums sind

- das Abiturzeugnis

- die Ergebnisse der Ersten Staats- (Lehrer-) Prüfung bzw. ihres praktischen Teils,

- Zeugnisse aus anderen Studienfächern,

- Thema und Aufbau der geplanten Dissertation,

- die Mitarbeit im Studium, insbesondere in Seminaren und an Forschungsvorhaben,

- die Bewährung bei der Leitung von Jugendgruppen und/oder die Mitwirkung in Turn- und Sportvereinen.

5. Die Entscheidung über die eingereichten Vorschläge trifft der Bundesauschuß für Wissenschaft und Bildung im Benehmen mit dem Wissenschaftlichen Beirat des DSB. 